\title{
Choix, décision et projet dans l'action : préséance du projet dans une perspective pluridisciplinaire
}

\author{
Jean-Pierre Boutinet ${ }^{1}$ et Jean-Pierre Bréchet ${ }^{2, *}$ \\ 1 Psychosociologie, Université catholique de l'Ouest, Angers, France \\ 2 Sciences de gestion, Université de Nantes, IAE Économie et management, Laboratoire d'économie et de management \\ Nantes-Atlantique, Nantes, France
}

Reçu le 27 octobre 2016. Accepté le 20 juillet 2018

\begin{abstract}
Alors que les grands enjeux auxquels nous sommes confrontés s'inscrivent dans des dynamiques complexes, caractérisées par les incertitudes, les ambiguïtés et des temporalités longues, les sciences économiques et sociales tendent à les aborder dans le cadre des théories de la décision « rationnelle». Cette situation paradoxale tient notamment au primat de l'économie dans nos sociétés. Elle tient aussi à la rareté des cadres d'analyse alternatifs qui permettraient de fonder des démarches d'anticipation et de gouvernance. L'ambition de cet article est de contribuer à combler ce manque en proposant une théorie de l'action collective fondée sur la notion de projet. Idée clé de cet article, une telle approche implique de s'interroger sur le sens de l'action et sur les valeurs, et s'inscrit dans des démarches qui interpellent directement l'interdisciplinarité.
\end{abstract}

La Rédaction

Résumé - Partant des fondements multidimensionnels de l'action, choix, décision et projet sont présentés comme trois grands modes de détermination des personnes et des groupes dans l'action. Ces trois modes n'ont pas la même importance ni la même place dans les grandes théories de l'action. Une explication majeure réside dans le traitement de la temporalité. L'action sociale comprise comme processus engage à poser la préséance du projet, porteur d'une temporalité englobante, au regard du choix et de la décision qui s'inscrivent dans des temporalités plus ponctuelles. Le concept de projet, qui trouve ses origines dans les sphères de l'architecture, de la philosophie, de la politique comme des sciences et techniques, témoigne aussi aisément de sa nature fondamentalement transdisciplinaire. Il semble à même de jouer un rôle important pour fonder une perspective pluridisciplinaire sur le plan paradigmatique et théorique comme sur celui des postures de recherche.

Mots-clés : action / temporalité / projet / choix / décision / théorie de l'action

\begin{abstract}
Choice, decision and project in action: precedence of the project in a multidisciplinary approach. Starting from the multidimensional foundations of action, choice, decision and project, these components are presented as the three main determination modes in action by individuals and groups. However, these three modes do not have the same importance or the same place in the main theories of action. A major explanation for this lies in the treatment of temporality/approach to temporality. Social action is understood as a process that involves putting precedence on the project, which carries an allencompassing temporality, with regard to choices and decisions that are part of more limited temporalities. The concept of project, with its origin in the spheres of architecture, philosophy, politics as well as science and technology, easily testifies to its fundamentally transdisciplinary nature. It appears to be able to play an important role in the founding of a multidisciplinary perspective at both the paradigmatic and theoretical levels, as well as with regard to the postures of research.
\end{abstract}

Keywords: action / temporality / project / choice / decision / action theory

\footnotetext{
*Auteur correspondant : jean-pierre.brechet@univ-nantes.fr
} 
Invitez un groupe d'étudiants ou d'auditeurs à identifier une décision personnelle importante pour leur vie, qu'ils auraient prise durant les derniers mois. Demandez-leur ensuite si cette décision était une bonne décision, voire la meilleure ${ }^{1}$. Le tour de table est instructif: « Je ne sais pas encore... Je vous répondrai dans quelques années... Oui, une bonne je crois mais la meilleure je ne sais pas, j'ignore ce qu'auraient donné les autres choix... Seul l'avenir le dira...». Les étudiants seront d'autant plus surpris du résultat du tour de table qu'ils auront suivi une formation à l'économie et que les problématiques du choix et de la décision auront été omniprésentes. Certains pourront toutefois avoir en mémoire ce qu'on leur a dit de la rationalité, toujours limitée, comme a su le montrer H. Simon, prix Nobel d'économie; ceci expliquerait que les choix et les décisions le soient aussi. La discussion tourne alors autour de la question de savoir si les étudiants ont été limités dans leur rationalité ou si, tout au contraire, ils ont eu de bonnes raisons de faire leur choix. L'édifice théorique construit sur les concepts de choix et de décision semble finalement bien fragile. Il est alors aisé de suggérer aux étudiants concernés qu'une lecture de leur façon de se déterminer, donc d'envisager les modes de détermination de l'homme dans ses façons de penser et d'agir, est de recourir au concept de projet.

Le concept de projet est ici compris, dans une perspective anthropologique, comme anticipation opératoire à caractère partiellement déterminé, ou de type flou, d'un avenir désiré (Boutinet, 2012). Figure de l'anticipation, la perspective opératoire indique que le projet ne peut porter sur le long terme trop conjectural ni se limiter au court terme trop immédiat. Son caractère partiellement déterminé fait qu'il n'est jamais totalement réalisé, toujours à reprendre, cherchant continuellement à polariser l'action vers ce qu'elle n'est pas. Enfin, la perspective d'un avenir désiré ou d'un idéal présuppose que le projet engage un auteur, individuel ou collectif, porteur d'une aspiration mobilisatrice et d'une énergie de changement. Le projet ainsi compris intègre les dimensions existentielles et opératoires de l' « agir » humain, non réductible au « faire » (Baechler, 2008).

Si l'on avance l'importance du projet dans l'action, notamment dans l'action collective, on observe par exemple dans la revue Natures Sciences Sociétés, que le concept apparaît de façon récurrente à propos de projets d'aménagement de territoires ou bien encore de projets de recherche pour lutter contre les effets du réchauffement climatique. On n'en est pas surpris, mais si l'on

\footnotetext{
${ }^{1}$ L. Bot et A. Solé à qui nous avions emprunté l'idée de cette entrée en matière il y a déjà de nombreuses années concluaient avec leurs étudiants qu'un humain ne peut prendre une « bonne décision». L. Bot et A. Solé, «Enseigner la contingence», http://ciret-transdisciplinarity.org/bulletin/b18c16.php.
}

traduit «projet» par project dans le monde anglo-saxon, le risque est grand qu'il y ait incompréhension avec le lectorat anglophone, car project renvoie au management de projet - project management- et non au concept de projet dans le sens anthropologique riche que nous privilégions ici.

Avancer le projet comme mode de détermination intentionnel des comportements en lien avec le choix et la décision communément employés n'est pas aisé. À l'instar du concept de projet, ceux du choix et de la décision ont connu de multiples appropriations et raffinements dans diverses disciplines (psychologie, économie, gestion, etc.) [Sfez, 1988]. Nous chercherons à montrer que les fondements constitutifs du modèle de détermination par le projet permettent de dépasser un certain nombre de limites des modèles du choix et de la décision, notamment celles tenant à la prise en compte de la temporalité. Les modèles du choix et de la décision apparaissent peu aptes à rendre compte de l'action humaine en tant que processus, et nous ajouterions volontiers avec A. Schütz (Schütz, 1998, p. 53), en tant que processus en cours conçu par l'acteur, c'est-à-dire en tant que conduite projetée. Ainsi, pour rendre compte de la façon dont les personnes ou les collectifs s'orientent et se déterminent, les modèles du choix et de la décision ne suffisent pas, ce qui ne veut pas dire que l'individu et les collectifs ne font pas de choix et ne prennent pas de décisions, mais ceux-ci gagnent à être envisagés en fonction d'un projet.

Nous construirons notre propos ${ }^{2}$ en rappelant d'abord que l'action est fondamentalement multidimensionnelle, avant d'envisager les modes de détermination que sont choix, décision et projet. À partir de l'établissement de la spécificité du projet, notamment de ses fondements anthropologiques transdisciplinaires, nous engagerons alors la réflexion sur la place du projet dans une perspective pluridisciplinaire.

\section{Les fondements pluridimensionnels de l'action}

L'action est par essence multidimensionnelle: elle engage des rapports à l'espace, au temps et aux acteurs et, sur ces trois dimensions d'inscription, de multiples aspects

\footnotetext{
${ }^{2}$ Cet article est le fruit d'une réflexion engagée depuis plusieurs années par ses auteurs, réflexion qui se nourrit de travaux de terrain menés à l'occasion de recherches collectives pluridisciplinaires, notamment dans le cadre du projet Claaq (Complémentarités locales pour l'accès à une alimentation de qualité). Ce projet de recherche-action a mobilisé l'association Bio Loire Océan et ses membres, à savoir le parc naturel régional, Angers Loire Métropole et des chercheurs en gestion, sociologie et économie (Bréchet et Dufeu, 2018).
} 
sont en jeu. C'est en ayant à l'esprit ce qui caractérise l'action mise en contexte, son caractère multidimensionnel et ses ancrages théoriques, que nous serons alors en mesure d'envisager choix, décision et projet comme trois modes essentiels de détermination de l'acteur agissant.

\section{L'inscription situationnelle de l'action}

De nombreux auteurs ont pointé combien l'action était incertaine dans son déroulement et son aboutissement, combien elle pouvait échapper à ses instigateurs et même conduire à des résultats contraires à ce qu'elle était sensée produire. E. Morin parle d'écologie de l'action dans ces termes (Morin, 2004, p. 234) ${ }^{3}$ :

«Du fait des multiples interactions et rétroactions au sein du milieu où elle se déroule, l'action, une fois déclenchée, échappe souvent au contrôle de l'acteur, provoque des effets inattendus et parfois même contraires à ceux qu'il escomptait.

$1^{\text {er }}$ principe: l'action dépend non seulement des intentions de l'acteur, mais aussi des conditions propres au milieu où elle se déroule.

$2^{\mathrm{e}}$ principe: les effets à long terme de l'action sont imprédictibles. »

On comprend immédiatement que s'intéresser à l'action, l'action que l'on peut qualifier de sociale, qu'elle soit individuelle ou collective, oblige à prendre la mesure de cette écologie généralisée de l'action et de la connaissance. L'écologie de l'action a pour pendant une écologie de la connaissance de même nature (Bateson, 1977 ; 1980). Les incertitudes qui frappent l'action sont aussi celles de la connaissance. Fort justement, la posture constructiviste en épistémologie retient que la construction de la connaissance et la construction de l'action sont liées, ce qu'E. Morin a mis au fondement de la pensée complexe qu'il défend (Morin, 1986).

L'inscription situationnelle de l'action, qui recouvre des actes concrets autant que des paroles, des connaissances disponibles autant que des ressources matérielles, engage des phénomènes d'interférence et d'interaction tant sur le plan cognitif (mésinterprétation, contradiction, opposition ou adhésion, etc.) que relationnel (dialectique de projets et de contre-projets, interdépendances stratégiques, jeux d'acteurs et phénomènes de pouvoir, etc.). L'action est régulation, pourrait-on affirmer dans une perspective inspirée de la sociologie des organisations (Crozier et Friedberg, 1977 ; Reynaud, 1997). Elle ne peut se comprendre en dehors de phénomènes d'autonomie et de dépendance qui caractérisent l'écologie généralisée que nous venons d'évoquer.

L'écologie généralisée de l'action et de la connaissance conduit à aborder l'action sur un mode dynamique

\footnotetext{
${ }^{3}$ E. Morin qui régulièrement parle de phénomènes d'auto-écoorganisation (Morin, 1977; 1980).
}

ou processuel, à envisager son déroulement en situation, et non à l'étudier isolément, encore moins statiquement. L'action est l'expression d'une capacité d'adaptation continue d'un acteur agissant en contexte, un acteur singulier avec ses propres justifications, ses capacités singulières d'accès aux ressources de toutes natures (savoirs, relations, ressources matérielles, etc.). Le contexte, toujours médiatisé par les perceptions que l'acteur s'en donne, est parfois contraignant, parfois facilitateur, et pose la question de l'accès aux ressources qu'il représente. On pressent immédiatement que l'intérêt porté à l'action en tant que processus ne peut s'épuiser dans des questions momentanées de décision et de choix ponctuels et, surtout, que la temporalité impliquée va jouer un rôle important dans une perspective nécessairement processuelle.

De ces points de vue, l'économie s'est bien plus intéressée à la décision et aux choix qu'à l'action. C'est pourtant un économiste, F. Perroux (Perroux, 1973), certes hétérodoxe, qui précise la compréhension de l'action sociale sur un mode qui nous convient parfaitement, en introduisant l'idée de projet:

«L'action sociale désigne les activités menées par des individus, groupes d'individus ou collectifs qui forment des projets, c'est-à-dire envisagent de façon anticipée, de façon plus ou moins rationnelle (c'est-à-dire réfléchie et adaptée) des fins et les moyens de les atteindre et qui, pour mener à bien ces projets, entrent en relation les uns avec les autres. L'action sociale produit des systèmes sociaux plus ou moins intégrés, emboîtés ou couplés. Elle met en jeu des phénomènes de pouvoir présents dans tous les domaines sociaux (religieux, politiques, intellectuels, etc.) et donc dans le domaine économique, des considérations d'intégration, d'appartenance, d'initiative ou de dépendance des agents et des interrelations entre les agents considérés comme des décideurs et des agents à projet. Les projets des décideurs se forment de façon plus ou moins intuitive ou calculée, compte tenu des relations et du pouvoir qui caractérisent ces relations » (Perroux, 1973, p. 77).

Cette longue citation exprime bien ce que met en jeu l'action, tant sur le plan individuel que collectif: des projets imbriqués, enchevêtrés dans leur conception et leur déploiement. Pas d'action sans interaction : 1'action s'exprime toujours dans un espace de sociabilité, et le contexte de l'action est forcément social. L'action est multidimensionnelle dans sa conception, dans sa construction et dans ses effets. Précisons ces caractères multidimensionnels.

\section{L'engagement pluridisciplinaire et pluriprofessionnel de l'action}

L'action est multidimensionnelle lorsqu'on la conçoit, lorsqu'on l'engage, lorsqu'on la mène. Dire cela affirme sa nature plurielle sur le plan de la 
connaissance et de la pratique en même temps que son caractère processuel.

L'action est éclairée par un champ pluridisciplinaire de connaissance puisqu'elle suppose, dans le souci de rationalité qu'elle implique, de tenir compte des rapports à l'espace, au temps et aux personnes qu'elle engage. Il ne s'agit pas de tenir ici un propos éthique, sans toutefois l'exclure, mais simplement de constater que le simple réalisme implique de prendre en compte des éléments qui tiennent aux acteurs de l'action et aux variables que celle-ci implique ou impliquera dans son élaboration et son déploiement. Les projets de lutte contre le réchauffement climatique et ses effets, qui sont emblématiques des recherches pluridisciplinaires actuelles, appellent, comme on le constate aisément dans les restitutions qui en sont faites, la convocation de divers univers disciplinaires (la climatologie, l'écologie, la géographie, la sociologie, le droit, etc.). Les projets de recherche qui précèdent ou accompagnent ces projets d'action nécessitent encore, à côté des savoirs académiques, les savoirs non académiques des citoyens et des politiques. Un numéro récent de la revue Futures (Lawrence, 2015) témoigne, à l'échelle internationale, de la nécessité d'éclairer l'action à concevoir ou à mener par une pluralité de disciplines.

La pluridisciplinarité s'associe toujours à un certain degré avec la pluriprofessionnalisation. L'action, dès lors qu'elle revêt une certaine envergure, est conduite dans un espace pluriprofessionnel. Cette idée est aisément admise. En effet, on ne peut postuler l'omniscience des auteurs / acteurs de l'action, pas plus que la polyvalence de leurs aptitudes pratiques. Il faut donc poser, a contrario, que l'action appelle la pluriprofessionnalité. Celle-ci est requise dès lors que l'action mobilise des expertises diverses, à la fois théoriques et pratiques. Pour ne prendre qu'un exemple, les projets d'aménagement urbain appellent non seulement des connaissances en urbanisme, géographie, architecture, mais requièrent aussi la collaboration effective de professionnels de ces univers disciplinaires qui interviennent à un stade ou un autre, avec leurs expertises propres, dans les réalisations concrètes que les projets prévoient.

Ainsi, à l'action en tant que processus, donc une action à concevoir et à conduire, font écho la pluridisciplinarité et la pluriprofessionnalité comme exigences, aussi bien sur le plan de la connaissance que de la pratique. Mais s'il faut penser l'action en situation, donc en tant que processus, comment théoriquement envisager ces exigences?

\section{L'action sous le regard théorique}

S'intéresser à l'action, c'est récuser le dualisme acteur-système, c'est-à-dire l'idée d'un acteur tout puissant et d'un système qui déterminerait les comportements. Cette idée, souvent reprise, bien établie (Crozier et Friedberg, 1977; Friedberg, 1993; Reynaud, 1997), oppose et repousse deux polarités interprétatives : d'un côté celle qui privilégie les acteurs et leur liberté, de l'autre celle qui ne considère que des déterminismes et des assujettissements. Elle est bien connue et largement dépassée par nombre de travaux. Retenons donc que l'action est le fait d'auteurs et d'acteurs agissant en fonction de quelque intention ou dessein, en situation d'appartenance à un ou des systèmes socioéconomiques, et donc de dépendance vis-à-vis d'échanges, de ressources et de circonstances. Les acteurs agissent en situation d'interaction idéelle et matérielle avec leur contexte d'action. Ayant indiqué précédemment l'importance d'une lecture dynamique ou processuelle de l'action, il nous faut introduire plus explicitement la question de la temporalité qui rythme cette action.

L'orientation temporelle de la causalité est importante pour comprendre l'opposition entre les polarités interprétatives que nous venons d'évoquer: «[...] la détermination par le passé contre la détermination par la visée intentionnelle d'un but et donc par l'anticipation $\mathrm{du}$ futur, et, dans les formes les plus réductrices de la polarisation, le mécanisme contre le finalisme » (Menger, 2001, p. 104). Les modèles déterministes relient causalement le présent au passé : par exemple, les théories déterministes en sociologie, sans qu'il s'agisse de schémas de conditionnement strict. P.-M. Menger évoque à ce propos le présent lourd des conditions du passé qui pèsent sur l'acteur et ses comportements dans l'habitus bourdieusien. Comme modèle opposé, ce même auteur pose la rationalité de l'acteur économique dans l'axiomatique néo-classique : «Les individus ont des préférences et des motifs d'agir (désirs, besoins); ils les connaissent parfaitement, ils cherchent à les satisfaire intelligemment sous la contrainte de leur budget, ils connaissent absolument la conséquence de leurs actes une fois ceuxci accomplis, et ils accomplissent dès lors ces actes à la lumière des conséquences qu'ils anticipent» (Menger, 2001, p. 136-137). De cette perspective conséquentialiste qui explique les comportements par les intentions et leurs conséquences anticipées, on peut dire qu'à bien des égards elle peut devenir aussi, paradoxalement, déterministe en théorie, dès lors que les individus sont interchangeables et optimisateurs. Car c'est bien l'acteur autonome et toutpuissant que l'on oppose à toute idée de domination du système et d'inféodation.

Dans les deux cas, l'action ne trouve pas sa place car, comme l'instruit très bien P.-M Menger, sont délaissées ses propriétés dynamiques et ses caractéristiques singulières liées à la différenciation des acteurs et des contextes d'action. Si l'action est l'objet à étudier, elle doit être comprise dans sa singularité, à l'aune des contraintes de toutes sortes qui pèsent sur elle. Elle doit l'être aussi à l'aune des orientations et finalités que lui donnent ses protagonistes. De ce point de vue, on peut 
s'associer avec A. Schütz (Schütz, 1998) pour dire qu'il s'agit de rechercher les «motifs-en-vue-de» (in order to motives) qui, du point de vue de l'acteur, réfèrent à son futur (l'acte projeté), tandis que les «motifs-parce-que» (because motives) expliquent l'action par les expériences passées qui à un degré ou un autre l'ont déterminé à agir (Schütz, 1998, p. 56). Les « motifs-en-vue-de» ont trait à l'acteur, à ses raisons d'agir dans le processus de l'action, ce que l'on retrouve au fondement de l'individualisme méthodologique. Le propre de l'individualisme est de reconnaitre l'action et le sens que lui donnent les acteurs, nous dit J.-D. Reynaud (Reynaud, 2003). Mais attention à ne pas assimiler, comme nous le rappelle cet auteur, la nature épistémologique et méthodologique du propos, qui reconnaît l'irréductible liberté de l'acteur avec, en pratique, ses marges de liberté effectives, qui peuvent être extrêmement restreintes.

On mesure à ces analyses l'importance du traitement de la temporalité et d'une analyse dynamique située, mais comment alors comprendre les figures de la détermination de l'acteur dans l'action, à savoir choix, décision et projet, dans ce qui les distingue?

\section{Le choix et la décision comme figures de la détermination ponctuelle}

Avec le choix et la décision, deux figures emblématiques de la détermination, notamment dans l'univers théorique de l'économie dont on sait l'importance, la dimension processuelle de l'action et l'action elle-même tendent à disparaître du fait d'un traitement du temps réduit à une succession de ponctualités. Ainsi, choix et décision, qui s'inscrivent dans une logique de la détermination ponctuelle ou de l'actualisation immédiate, vont être associés et distingués du projet, mode de détermination fondé sur l'anticipation de type flou et qui engage des lignes de conduite processuelles et des actualisations différées.

L'action de choisir relève d'une détermination par laquelle l'acteur concerné donne la préférence à une option, une possibilité, en écartant les autres. Il ne peut y avoir de choix que s'il y a liberté et pouvoir d'opter entre différentes solutions. L'acteur a-t-il le choix si se présentent à lui, sous la contrainte par exemple, deux propositions entre lesquelles il doit se déterminer, sans qu'aucune ne lui agrée vraiment? Si c'est le cas, on mesure bien que l'on a affaire à une acception problématique de la notion de choix, du fait qu'il n'y a pas réellement préférence. Le concept de choix présuppose ainsi un ensemble de possibles parmi lesquels on va en retenir un ou plusieurs. Pour que le choix puisse s'exercer, il faut qu'existe un ensemble de possibilités identifiées ou identifiables entre lesquelles opter. Le dictionnaire rappelle opportunément que le mot choix désigne un ensemble d'options parmi lesquelles on peut se déterminer, c'est-à-dire un existant de propositions (assortiment, collection, éventail, gamme, etc.) qui se présentent à l'acteur qui va choisir. Le choix exprime une préférence; une élection est possible, mais cette élection n'a de sens que si une sélection ou une identification sélective peut être opérée au préalable dans un référentiel ou un registre. Le choix ne peut être pensé en dehors d'objets désignés, identifiables. De ce point de vue, les choix de projets ne peuvent être assimilés à des choix d'objets. On comprend alors la situation limite pour ne pas dire l'aporie qu'il y a par exemple à parler de choix d'un avenir lorsque les avenirs sont plus à construire qu'à choisir parmi un éventail d'avenirs identifiés dans leurs divers registres constitutifs ${ }^{4}$. Nous reviendrons plus loin sur ce point.

Si l'on retient enfin que le choix, c'est aussi l'ensemble des choses retenues, on mesure alors les liens qui existent entre choix et décision. La décision est le fait d'un acteur - individuel ou collectif- qui effectue délibérément un choix entre plusieurs solutions susceptibles de résoudre le problème ou d'améliorer la situation à laquelle il s'estime confronté. On retrouve, avec cette acception de la décision, la première compréhension du mot « choix » comme action d'opter et donc de se décider. Pour qu'il y ait décision, il faut qu'il y ait choix, donc préférence, sélection et possibilité du choix effectif, avant que la décision ne vienne trancher. H. Simon avait souligné ces liens forts entre choix et décision en faisant observer que lorsque l'on fait intervenir les aspects de perception et de cognition dans le processus de décision, il faut prendre en compte le processus de choix (Simon, 1959, p. 272). Le processus de décision ne peut se penser sans introduire ce qu'il implique de préférence et de sélection et donc de considérations ayant trait au choix, à l'identification des choix possibles et à l'action même de choisir, donc de se décider, tant sur le plan individuel que collectif. Mais conformément à l'étymologie du mot «décision», du fait de l'importance de l'idée de rupture ou de scission ${ }^{5}$, la décision marque un avant et un après, signifie un engagement. La décision s'apparente à un engagement tant vis-à-vis de soi-même que de ses interlocuteurs. De ce point de vue, s'il existe un processus de prise de décision, il n'y a pas de processus proprement dit de décision: la décision est prise ou elle ne l'est pas.

\footnotetext{
${ }^{4}$ Nous préférons ici utiliser le terme «avenir» de préférence à son synonyme «futur», car l'avenir définit le monde des possibles qui s'offrent à l'acteur, alors que le futur en tant que «devoir être» renvoie à l'inéluctable auquel on ne peut échapper.

${ }^{5}$ L'étymologie de «décision» est de ce point de vue suggestive, qui fait dériver le verbe «décider» du latin caedere, signifiant couper, trancher, une fois retiré le préfixe «de».
} 
Ainsi, si les interprétations processuelles de la prise de décision présentent un intérêt, elles ne nous semblent pas à même d'introduire la temporalité de façon satisfaisante dans l'art de se déterminer. Celle-ci demeure une temporalité de la ponctualité plus que de la continuité. Les phénomènes d'apprentissage n'y trouvent pas leur place, alors qu'ils sont au fondement de l'action. Une illustration de l'insuffisance, voire de la disqualification, du paradigme du choix, constitutif de celui de la décision, se manifeste aisément dans le contexte des démarches de conception innovante. Concevoir en entreprise un nouveau médicament ou un nouvel ordinateur, ce n'est évidemment pas choisir entre différentes éventualités préexistantes parfaitement identifiées. Il s'agit, par toute une série d'itérations et d'arbitrages, d'invention et de conception mêlées, de produire un objet nouveau, un inédit. Celui-ci n'a pas été choisi à proprement parler. Dans les démarches entrepreneuriales, et nous évoquerons plus loin la création d'une association originale et innovante de producteurs biologiques de fruits et légumes, ces propos qui privilégient la conception sur le choix trouvent aussi toute leur pertinence : les producteurs ont innové en créant des solutions originales pour faire vivre leur projet associatif singulier. Dans tous les cas, on pourrait dire que le «chemin se fait en marchant» pour rendre compte que l'action se nourrit des apprentissages qu'elle produit. J.-D. Reynaud exprime ces idées en comprenant l'action comme régulation, ce sur quoi nous aurons l'occasion de revenir: "L'acteur social ne se borne pas à choisir la meilleure éventualité, il invente des solutions. Il ne se borne pas à choisir le meilleur coup à jouer et la meilleure stratégie dans un jeu social, il maintient ou transforme les règles du jeu » (Reynaud, 1991, p. 14). Cette approche par la régulation conduit l'effort de théorisation vers des perspectives processuelles inhérentes à tout compréhension de l'action collective comme apprentissage (Hatchuel, 2000 ; 2005), en rajoutant la nécessité de saisir l'émergence de l'action. L'action a des auteurs et elle naît d'un projet.

\section{Le projet dans l'action: de la détermination de l'acteur à la conduite de l'action}

«Choisir parmi des projets d'action», tel était le titre d'un article d'A. Schütz de 1951 qui s'inscrit pleinement dans la perspective de notre propre propos (Schütz, 1998). Les choix de projets ne sont pas des choix d'objets nous dit-il. Choisir parmi des projets apparait comme un abus ou une facilité de langage sans réel fondement théorique/scientifique, puisque les conditions qui permettent de penser le choix ne sont pas réunies : les futurs ou avenirs ne sont pas connus par anticipation, ne sont pas des objets à la portée de l'acteur; tout au contraire, l'acteur participe à la construction de l'avenir qui se présentera au fur et à mesure du déroulement du temps. Les choix d'objets confrontent à des objets qui coexistent et sont accessibles dans le temps présent, alors que les projets d'action future ne coexistent pas dans la simultanéité du temps externe à l'acteur (Schütz, 1998, p. 74). Envisager le projet dans l'action conduit bien à s'éloigner radicalement de la détermination par le choix et la décision.

Retenons la définition du projet comme anticipation à caractère opératoire de type flou d'un avenir désiré (Boutinet, 2012). Que le projet soit une anticipation opératoire indique qu'il ne saurait se réduire à un simple acte d'imagination plus ou moins spéculative ou divinatoire. La préoccupation anticipatrice va toujours de pair avec le souci de l'actualisation du projet : le projet esquissé vise en même temps une réalisation effectivequi le rendra manifeste et ce faisant assurera une transition depuis la sphère de la virtualité vers celle de l'actualisation. Cette double face anticipation-actualisation est constitutive de l'activité projective. Que le projet représente une anticipation de type flou le distingue du plan, du but ou de l'objectif qui sont de type déterministe et techniciste. Le projet, comme détermination personnelle, ne saurait relever du déterminisme ni de la fixité. Dans le contexte de l'écologie généralisée de la connaissance et de l'action, il s'inscrit dans les temporalités marquées par les incertitudes, les aléas, les effets de composition ou de systèmes inattendus (effets d'agrégation, effets pervers, interdépendances cachées, émergences de propriétés nouvelles, etc.). L'auteur du projet lui-même évoluera dans ses préférences et ses aptitudes propres au fil du temps. L'action est apprentissage. Si l'on introduit enfin la perspective d'un avenir désiré, d'un idéal ou d'une aspiration mobilisatrice, l'intention de réaliser le projet suppose de tenir compte de la réalité des possibilités de faire vivre le triptyque aspirationanticipation-actualisation.

Avec le projet, il s'agit donc de fonder, en raison, en intelligibilité mais aussi en pratique dans la construction de l'action, la possibilité d'une action à travers la concrétisation au cours du temps d'une intention, d'endsin-view dirait encore J. Dewey. Ceci implique l'idée d'une certaine planification porteuse de lignes de conduite qui représentent des économies de délibération et de coordination, tant avec soi-même comme auteur qu'avec les autres, ce qu'a su montrer la philosophe analytique de l'action (Dupuy et Livet, 1997). Les auteurs de cette philosophie parlent certes d'action planifiée et non projetée, notamment M. E. Bratman (Bratman, 1997) qui envisage une théorie modeste de l'action planifiée avec les avantages qu'elle présente d'un point de vue rationnel.

On voit en conséquence du type de compréhension du projet que nous venons d'exposer que la détermination 
par le projet se distingue bien des déterminations par le choix et la décision, non seulement en tant que mode de détermination à proprement parler - dans la sphère de la virtualité comme figure de l'anticipation-, mais aussi en tant que détermination continuée dans la conduite de l'action effective qui se déroule-comme processus d'actualisation. Mobiliser le projet, c'est toujours à un degré ou un autre s'engager dans le double défi de mener à bien un travail de conception que l'on a initié et de conduire à son terme le travail de réalisation subséquent dans une situation marquée par la complexité, l'incertitude et la singularité. La spécificité du concept de projet tient particulièrement à la prise en compte des temporalités de la continuité qu'il intègre, temporalités de l'anticipation dans son ébauche, temporalités processuelles dans sa mise en œuvre, alors que le choix et la décision ne peuvent envisager la temporalité que sur le mode exogène de la simultanéité et de l'irréversibilité.

\section{Le projet au fondement de la pluridisciplinarité}

Après avoir présenté choix, décision et projet dans ce qui fait la spécificité de chacune de ces déterminations de l' « agir », il nous faut maintenant justifier de la préséance du projet dans l'action. Concept fondamentalement transdisciplinaire, le projet est à même de saisir les fondements multidimensionnels que nous avons précédemment posés, tant du point de vue de la connaissance de l'action qu'à propos de l'action elle-même. Pour cette raison, le projet est au fondement de la pluridisciplinarité, ce dont témoignent les pratiques de recherche soucieuses de produire des savoirs pour l'action.

\section{Un concept transdisciplinaire pour une préséance dans l'action}

L'usage du substantif " projet» commence à s'imposer à la Renaissance. Dérivé du déverbal «projeter», luimême issu du latin projicere, indiquant l'action de jeter en avant, le projet signifie l'idée que l'on extériorise, que l'on avance, ainsi que le plan conçu pour réaliser cette idée. Le concept de projet se constitue au fil du temps autour de plusieurs dimensions structurantes qui l'ancrent dans la transdisciplinarité :

- la dimension biologique, qui associe le projet au vivant et à l'inédit pour conjurer la répétition mortifère;

- la dimension philosophique, notamment existentialiste et phénoménologique, qui voit dans le projet un mélange d'intention et d'anticipation qui donne sens à une existence, celle de son auteur;

- la dimension politique et culturelle, qui valorise dans les espaces modernes progrès et projet, amenant par là les collectifs humains à affirmer leur volonté de maitriser leur devenir;
- la dimension pragmatique, historiquement la première à se manifester à travers le projet architectural, qui, à travers l'ingénierie de projet, cherche à singulariser la conception et la fabrication des différents dispositifs et objets techniques ou industriels.

Chacune de ces dimensions incarne l'une des propriétés du projet comme antidote susceptible de conjurer ce qui constamment le menace : l'inédit du vivant entend conjurer la répétition et la mort; l'innovation cherche à le prémunir contre les risques d'enfermement et de régression; l'anticipation méthodologique se pose en opposition à l'impulsion et à l'improvisation; la recherche de sens de l'existence vise à défier le hasard et l'absurde.

Ces dimensions fondatrices de la compréhension anthropologique du concept vont se retrouver dans la mobilisation du terme. Mais l'omniprésence du projet dans nos sociétés, parfois dites «de» ou «par» projet (Boltanski et Chiapello, 1999), s'avère indissociable d'une multiplicité d'emplois du mot sur un mode ambigu au regard de la constitution historique que nous venons d'évoquer: projet d'objet, projet d'établissement ou d'entreprise, projet associatif, projet de changement, projet d'orientation, projet professionnel, projet politique, etc. Il reste néanmoins que mobiliser le projet, ou en regretter amèrement son absence dans la sphère sociétale ou dans la sphère politique, aussi bien d'ailleurs à l'échelle nationale qu'européenne, c'est par le fait même affirmer en même temps son importance pour fonder l'action. Un ballotement sans perspective est mal vécu; il n'offre pas de perspective qui fasse sens.

Dès lors que l'on délaisse les seuls projets techniques pour envisager les projets aux dimensions existentielles, qu'ils soient individuels ou collectifs ${ }^{6}$, on ne peut penser le projet sans l'associer à un auteur déterminé. Le projet engage une pronominalisation, un «je » ou un «nous». On le comprend aisément à l'échelle individuelle. L'échelle collective pose la reconnaissance de l'auteur comme problématique, voire comme problème. La dimension existentielle, qui est aussi celle du sens, prend une grande importance dès lors que le projet est posé comme constitutif du collectif (Desreumaux et Bréchet, 2009; Reynaud, 1997). L'action engage plusieurs compréhensions du sens, mêlées dans les considérations existentielles et opératoires de tout projet :

- le sens-orientation de l'action envisagée, direction à emprunter, orientation dans l'espace et le temps, perspectives précisées à l'horizon du projet;

- le sens-signification de l'action anticipée par le projet, signification apportant en termes de motifs une

\footnotetext{
${ }^{6}$ Mais toujours collectifs et individuels à un degré ou un autre : les projets dits individuels impliquent toujours des collectifs encadrant et les projets collectifs s'appuient toujours sur des acteurs/auteurs individuels qui jouent un rôle moteur ou catalyseur.
} 
justification, une rationalisation à l'action à entreprendre et donc déniant à cette action la possibilité d'être gratuite ou inconséquente, voire absurde;

- le sens-pertinence de l'action à entreprendre présentement, sur un mode approprié au regard du contexte environnemental au sein duquel l'acteur envisage de déployer son projet pour initier un imprévu, bousculer une situation, créer un inédit.

Le projet, concept transdisciplinaire, assure, par le(s) sens qu'il engage, une préséance dans l'action. Attaché à un auteur, il a toujours à voir, au présent, avec un itinéraire déjà constitué, un passé à élucider pour penser un renouveau désiré, pour greffer un inédit anticipé. Le passé s'inscrit dans la singularité d'un rapport aux personnes, à l'espace et au temps, un passé entrevu en continuité ou en rupture avec le moment présent ou celui à venir. Et cette inscription fondamentale explique la préséance du projet au regard de l'action à venir (Boutinet et Bréchet, 2014). On ne saurait dire que le choix et la décision ignorent ces aspects dans les procédures, voire les processus d'instruction et de délibération qu'ils recouvrent. Mais le projet, toujours singulier, attaché à un auteur, ne peut rester dans la simplification, encore moins dans la caricature. Le sens, à la fois orientation, signification et pertinence, est engagé dans la légitimité de l'action collective à mener, en vue d'un mode de détermination validé socialement. La figure du projet renvoie en effet à un mode de détermination socialisé, qu'il s'agisse des projets individuels ou des projets collectifs. Le projet connaît bien sûr l'épreuve de l'action qui le valide ou l'invalide pour tout ou partie de ce qu'il prévoyait, cette épreuve permettant de lui attribuer une reconnaissance sociale du travail de conception dont il est le produit. N'est-ce pas alors le lien avec la recherche, et plus justement les liens recherche-action, qui se trouvent associés à ce concept profondément transdisciplinaire?

\section{La posture du chercheur: projet de recherche et projet d'action}

G. Bachelard, dans la fulgurance coutumière de ses propos, place immédiatement le projet au fondement de la recherche: «Au-dessus du sujet, au-delà de l'objet, la science moderne se fonde sur le projet. Dans la pensée scientifique, la méditation de l'objet par le sujet prend toujours la forme du projet » (Bachelard, 1934, p. 15). On sait que la posture constructiviste en épistémologie met en son cœur les projets de connaissance, que l'on nourrit en fonction des projets d'action envisagés. Dès lors, la pertinence de la connaissance produite tient à la capacité de celle-ci d'instruire l'action pour agir en plus grande sûreté intellectuelle. Qu'est-ce alors qu'une bonne théorie? Réponse de J. Dewey:
«La théorie, lorsqu'elle est séparée de l'agir et du faire concrets, est vide et vaine ; [...] Le problème de la relation de la théorie à la pratique n'est pas qu'un problème théorique. C'est aussi le problème le plus pratique auquel nous ayons à faire face dans la vie. Un problème qui soulève la question de savoir comment l'intelligence peut informer l'action et comment l'action peut permettre une compréhension plus grande de la signification: une perception claire des valeurs dignes d'intérêt et des moyens grâce auxquels on pourra les garantir par des objets dont il est fait l'expérience. La construction d'idéaux en général et leur glorification sentimentale sont aisées; en rester là, cependant, revient à éluder nos responsabilités en matière de pensée et d'action rigoureuses » (Dewey, 2014, p. 297).

La recherche soucieuse de s'ouvrir aux multiples aspects de l'action en situation (espace, temps, acteurs) est une recherche ouverte à la pluridisciplinarité et à la pluriprofessionnalisation, à l'instar de l'action ellemême. Le numéro spécial de Futures «Advances in Transdisciplinarity» de 2015, dont la revue Natures Sciences Sociétés a proposé la recension (Bréchet et Gigand, 2016), témoigne bien du fait que les recherches dites transdisciplinaires recouvrent essentiellement des protocoles de recherche-action impliquant diverses parties prenantes académiques et non académiques -acteurs politiques et sociaux au sens large-, ainsi que divers champs disciplinaires, autour d'objets de recherche qualifiés de complexes à de nombreuses reprises, avec le souci affirmé d'engager des actions de changement ou de transformation. Y sont directement posées les questions des collectifs d'acteurs de ces projets de recherche et d'action, de l'association fructueuse des disciplines, de l'apprentissage commun des acteurs impliqués, de l'intégration des savoirs et pas seulement leur juxtaposition, de l'implication incontournable encore des acteurs non académiques dans les protocoles. Comment ne pas discerner, dans ces attributs de la pluri- et de la transdisciplinarité, les ingrédients caractéristiques de la détermination par le projet?

Une pertinence scientifique se joue dans la reconnaissance de la place du concept de projet. Dès lors que l'on se préoccupe d'action et de changement, la pluridisciplinarité s'invite, et avec elle le projet, pour penser et mettre en œuvre le souhaitable. En même temps, s'affirme la posture clinique de production de connaissance qui engage enquête et expérimentation (Dewey, 2014). On ne peut s'en tenir aux théories générales et à leurs arguments, sans pour autant récuser l'intérêt de ceux-ci. Le caractère scientifique repose ici sur la capacité d'interagir avec le terrain des pratiques pour produire une connaissance pertinente eu égard aux projets d'action nourris. La robustesse scientifique se joue dans la méthode plus qu'au niveau de la valeur de généralisation des résultats obtenus (Friedberg, 1993). Soucieux de validité procédurale plus que substantive, le 
projet de connaissance et d'action est au fondement d'une approche pluridisciplinaire pour orienter une action inscrite dans sa pluridimensionnalité.

\section{La perspective d'une théorie de l'action collective fondée sur le projet}

L'action collective, quelle qu'elle soit, engage une capacité d'agir en commun qui suppose un projet collectif. Dit autrement, les collectifs naissent d'un projet imaginé, conçu et concrétisé. Mais comment, en théorie, établir le lien entre le concept de projet et la régulation de l'action? Plus précisément, nous devons nous interroger sur la manière dont l'effort de théorisation de l'action mobilise le projet pour comprendre le passage de la sphère de la virtualité à celle de l'actualisation. Certes, l'action collective se fonde sur un projet collectif compris comme anticipation opératoire, mais elle n'existe que si ce projet engage une possibilité d'action commune fondée sur des règles explicitées et vécues, génératrices d'apprentissages collectifs. L'action collective, qui s'inscrit dans la durée, recouvre une régulation qui engage à un degré ou un autre le devenir commun d'un collectif. Le projet collectif, en tant qu'ensemble de règles que se reconnaît le collectif et qu'il fait vivre, fixe ses propres frontières et contribue à sa propre identité (Reynaud, 1997).

Cette perspective, d'inspiration sociologique, devrait aussi faire sens en matière de théorie de l'entreprise et être largement acceptée par les sciences sociales, et notamment en économie. Mais les théories dites de la firme, d'inspiration économique, ne traitent pas directement de l'entreprise réelle - l'organisation productiveet de ses préoccupations de management. La théorie de l'agence ou la théorie des coûts de transaction, qui privilégient les facettes d'allocation, d'efficience et de coût, délaissent largement les dimensions de conception et de production collective de l'action. Les théories qualifiées d'évolutionnistes, qui portent leur attention sur les compétences et les connaissances des entreprises, nourrissent une perspective plus riche mais n'épuisent pas les questions d'émergence des organisations ni celles ayant trait, par exemple, aux questions de politique générale au sens large (orientations, valeurs ou principes privilégiés).

Proposer une théorie de l'entreprise fondée sur le projet revient à comprendre l'action collective comme expression d'un projet collectif à la fois politique, économique et organisationnel (Bréchet et Desreumaux, 2012 ; Desreumaux et Bréchet, 2009; 2018). Considérer l'entreprise sur cette base revient à instruire les questions des «pourquoi», des «quoi» et des «comment» de l'action, qui se trouvent au fondement de la constitution des collectifs, ce qui ne revient pas à nier ou exclure les arguments de coûts de transaction, de ressources, de routines ou d'apprentissages habituellement mobilisés. Mais l'effort de théorisation nécessite d'associer ces arguments à l'existence d'un projet collectif. Cette compréhension de l'entreprise comme projet est d'application générale à toute forme d'action collective. L'exemple, présenté ci-dessous, d'une association de producteurs de légumes et fruits biologiques ${ }^{7}$ illustre bien, nous semble-t-il, la centralité du concept de projet dans toute compréhension, émergence et constitution de l'action collective.

En 2017, l'association Bio Loire Océan (BLO) compte soixante-sept adhérents producteurs de fruits et/ou légumes bio, situés dans les cinq départements des Pays-de-la-Loire, majoritairement dans la LoireAtlantique (44) et le Maine-et-Loire (49). Dans leur ensemble, les adhérents de BLO couvrent plus de 220 hectares de vergers, 270 hectares de maraîchage de plein champ et 15 hectares de surface couverte, ce qui représente environ le quart de la production régionale en fruits et légumes bio, pour un chiffre d'affaires de 18 millions d'euros environ. L'activité commerciale et les débouchés, principalement avec le réseau Biocoop, représentent un aspect dont il faut bien évidemment reconnaître l'importance pour la viabilité du collectif de producteurs et de chacun d'entre eux. Mais tous ces producteurs doivent réaliser une part majoritaire de leurs ventes en dehors du circuit associatif afin de maintenir l'indépendance de chacun. Les débouchés des membres pour les ventes hors BLO sont variés, soit en circuits courts comme l'exige le cahier des charges de l'association (Amap ${ }^{8}$, ventes à la ferme, magasins de producteurs, etc.), soit en circuits longs (grossistes, réseaux, grande distribution, etc.). Mentionnons aussi le fait que $10 \%$ des adhérents ne font pas de commerce via l'association, certains venant y chercher avant tout les échanges avec des pairs ou la participation à des programmes particuliers, comme par exemple au programme d'action sur les semences paysannes, qui a été engagé par BLO en 2006, en collaboration avec des chercheurs. Pour d'autres adhérents enfin, il s'agit surtout de marquer leur adhésion au projet associatif d'ensemble.

Les textes de référence que sont la charte et le cahier des charges (consultables en ligne ${ }^{9}$ ) expriment les valeurs et les objectifs partagés par les producteurs qui, pour la plupart, ont délaissé les coopératives dont les fonctionnements ne leur convenaient plus. La charte, qui affirme les valeurs écologiques, économiques, sociales et

\footnotetext{
${ }^{7}$ Un long travail de recherche-accompagnement (Bréchet et al., 2014) a été mené dans la durée sur cette association avec notamment Ivan Dufeu, professeur à l'université d'Angers et Nathalie Schieb-Bienfait, maître de conférences à l'université de Nantes. Qu'ils soient ici remerciés pour leur contribution. ${ }^{8}$ Associations pour le maintien de l'agriculture paysanne.
} 
sociétales, se précise dans le cahier des charges. En cela, bien sûr, cette charte qui associe des exigences de nature fort différentes, s'affirme comme multidimensionnelle et l'on pourrait dire «multivalorielle». Le cahier des charges, d'une dizaine de pages, recouvre quant à lui les critères qui précisent la charte, c'est-à-dire les exigences que les pratiques du collectif et de ses membres doivent respecter. Le respect de ces critères est observable et potentiellement contrôlable. On peut ainsi considérer que les textes de référence, élaborés et précisés par les producteurs au fil du temps, sont l'expression du projet de référence de $\mathrm{BLO}$, en tant qu'anticipation à visée opératoire $^{10}$. Ils explicitent en effet les règles dont le collectif s'est doté et qui sont à prendre en compte par lui dans ses pratiques professionnelles, la règle étant, comme le projet, une figure de l'anticipation opératoire : nous comprenons ici le concept de règle comme articulant logiquement des principes et des critères régulateurs de l'action. Mais le projet, aussi bien que la règle, ne saurait en rester à l'ordre du texte et de l'énoncé. En pratique, et la pratique de BLO en témoigne aisément, il s'agit de les faire vivre aussi bien dans les pratiques de gouvernance que dans celles de production et de commercialisation. Un apprentissage est en jeu: des modalités de coordination se sont améliorées, des outils ont été développés, de la confiance est née dans les échanges; les résultats obtenus, parfois bons, parfois mauvais, ont validé ou invalidé certaines orientations. Aborder les pratiques serait ici bien trop long et d'un apport limité pour notre propos, puisqu'il ne s'agit pas de les décrire ni de les caractériser. Mais en pointer l'importance vise à rappeler que, dans une théorie de l'action, le projet et les règles n'existent qu'en tant qu'ils s'actualisent dans les pratiques. À propos de la règle, dans la perspective philosophique qui est la sienne, J.-P. Cometti (Cometti, 2011) insiste sur le fait qu'elle n'existe pas dans la sphère des idées mais bien en tant qu'elle est appliquée, et de plus correctement appliquée ajoute-t-il. Cette position vaut pour le projet. Les pratiques, c'est-à-dire les régulations effectives, font vivre le projet et les règles, avec leurs réussites, leurs difficultés et parfois leurs échecs. Le projet dans lequel les producteurs retrouvent leurs aspirations ne tiendrait pas longtemps le collectif si les pratiques n'en respectaient pas les exigences ni n'en prouvaient la viabilité.

Une communauté de projet, si elle engage la perspective d'un monde commun (Reynaud, 2008), un avenir désiré (Boutinet, 2012) ou un idéal imaginé

\footnotetext{
${ }^{9}$ www.bioloireocean.bio/medias/site-25/Charte $\% 20$ Bio $\%$ 20Loire\%20Ocean.pdf.

${ }^{10}$ Sans oublier l'importance des dispositifs de la médiation marchande que nous délaissons ici, à savoir notamment la planification des cultures, la facturation centralisée et l'offre en ligne.
}

(Dewey, 2011; 2014), se comprend d'abord comme une communauté de règles vécues, et comme une communauté d'apprentissage qui fonde une capacité d'action commune. Les valeurs importent, les acteurs peuvent s'y référer, elles peuvent être l'objet de discussions et de controverses, comme c'est bien sûr le cas chez BLO. Mais l'action collective se comprend en ce qu'elle engage des règles, plus justement une régulation autour d'un projet qui la cadre par ses règles. L'apprentissage que recouvre l'action collective n'exclut nullement les phénomènes de négociation et de pouvoir constitutifs des échanges sociaux.

Cette compréhension d'ensemble est au fondement de la théorie de la régulation sociale de J.-D. Reynaud. L'action collective qui produit ses propres règles n'existe qu'en tant qu'elle fait vivre ces dernières dans et par la régulation du collectif. C'est notamment par le travail de régulation que l'action collective requiert, que se joue l'intégration de l'acteur collectif, avec ses membres dans leur diversité de statuts, d'engagements et de logiques de participation. Les acteurs de BLO font des choix, prennent des décisions, mais c'est en référence à un projet qui rend l'action intelligible et participe à sa construction. Connaissance et action sont associées, et ce lien fort se retrouve au fondement du pragmatisme dont on sait l'importance qu'il accorde à l'action réfléchie en situation, l'action avec ses difficultés, ses contingences et ses incertitudes, qui expliquent, selon J. Dewey, que bien des penseurs aient privilégié la connaissance épurée: « La certitude parfaite, voilà ce que l'homme veut. Or on ne peut la trouver dans l'action et la fabrication pratiques. Celles-ci se produisent dans l'horizon d'un avenir incertain, et impliquent des périls, le risque de mésaventures, la frustration et l'échec » (Dewey, 2014, p. 40).

\section{Conclusion}

S'engager dans l'action, expérimenter et apprendre dans l'action, ne peut se penser sans le recours au concept de projet qui anticipe, dynamise et oriente celle-ci. Les collectifs naissent de projets, se construisent par les projets qu'ils conçoivent et qu'ils font vivre. Bizarrement, le terme de projet dans le sens où nous l'avons compris se traduit mal dans le monde anglo-saxon, qui parle plus volontiers de "program», et souvent de «project» au sens du management de projet. Mais des sociologues anglophones ont aussi bien compris la nécessité de mobiliser le projet dans le sens riche et transdisciplinaire que nous avons retenu (Emirbayer et Mische, 1998).

Le concept de projet demande à être habilité dans sa spécificité eu égard aux concepts de décision et de choix. Il faut affirmer, pensons-nous, la préséance du projet sur ces autres modes de détermination que sont le choix et la 
décision qui, peuvent, eux, être largement rapprochés l'un de l'autre. (Ré)habiliter la place du projet dans une théorie de l'action, c'est reconnaître l'importance de l'«agir» projectif dans son mode itératif, soucieux d'articuler anticipation et réalisation pour fonder une théorie de l'action. Aussi bien à l'échelle individuelle que collective, il s'agit d'un "agir» englobant compris comme un «agir» créatif (Joas, 1999) d'anticipation associant des considérations axiologiques et instrumentales. Cet « agir » engage les idées, les valeurs, les intérêts (Kalberg, 2010), mais aussi les considérations d'anticipation, de planification et de mise en œuvre inhérentes à la conception et au déploiement de l'action. Il faudrait ajouter par ailleurs que l'on doit prendre la mesure de l'importance des émotions (Damasio, 1995), des effets de mémoire et d'anticipation (Berthoz, 2003; Berthoz et Debru, 2015), liés au fonctionnement du cerveau, et constitutifs de l' « agir » projectif(Bréchet et Desreumaux, 2010). Dans cette perspective, les modèles du choix et de la décision peuvent se présenter séparément comme des variantes isolées ou des moments isolés dans l'art de se déterminer, au regard du projet, mais c'est bien par rapport à un projet que les choix et les décisions prennent sens.

$\mathrm{Ce}$ caractère englobant du projet nous guide vers des questions mal traitées par la décision et le choix. Si ces derniers modes de détermination prédominent, sans projet pourrait-on dire, alors se pose la question du sens, en même temps que s'exprime une forme d'irrationalité ou d'inconséquence. L'«agir» projectif comme mode d'expression typiquement humain est menacé en permanence par les exigences présentistes de nos environnements postmodernes qui privilégient le profit immédiat, réduisent l'espace au territoire avec ses ressources à exploiter, et menacent les acteurs dans la singularité de leur vie (Boutinet et Bréchet, 2014). Les collectifs et les personnes vivent alors le ballotement qui va de pair avec la vulnérabilité et l'assujettissement aux dominations d'un moment présent court-termiste, celui de l'immédiateté et de l'urgence qui écrasent toute autre considération d'émancipation. Ce n'est pas pour rien si les collectifs en appellent au projet, car celui-ci peut à bien des égards être considéré comme le bien commun des collectifs (Desreumaux et Bréchet, 2013). Mais s'il est dans l'ordre des choses que le projet soit convoqué lorsqu'un acteur individuel ou collectif fait face à des situations qui ne sauraient le laisser indifférent, ceci explique vraisemblablement aussi le cercle vicieux dans lequel nos sociétés se laissent enfermer. En effet, la frénésie de projets que nous observons aujourd'hui dans la multiplicité plus ou moins désordonnée des initiatives prises génère de plus en plus souvent des projets factices qui agacent autant les acteurs qui les réalisent que ceux qui les évaluent.

En guise de perspective à la présente réflexion, synthétisons notre propos autour des conditions d'éta- blissement d'une théorie de l'action fondée sur le projet. Sur le plan individuel, il faut reconnaître l'acteur, dans ses fondements anthropologiques, comme un auteur porteur d'une œuvre à concrétiser, et son " agir » projectif comme un « agir» créatif à la fois d'anticipation et de concrétisation, oscillant entre rationalité axiologique et rationalité instrumentale. Sur le plan collectif, groupal ou entrepreneurial, le projet collectif constitue une figure d'intégration des collectifs de travail par le jeu des régulations de l'action anticipée et déployée. Le jeu des régulations donne aussi tout son sens à l'art de la négociation, promotrice du lien social porté par tout projet collectif. De ce point de vue, nous pouvons aisément mesurer ici la proximité de perspective avec la théorie de la régulation sociale de J.-D. Reynaud qui met en son cœur la préséance logique de la prise en compte de l'action collective pour appréhender les phénomènes sociaux. Enfin, comment ne pas voir la pertinence, pour aborder les organisations humaines, des appropriations du concept de régulation dans les diverses disciplines? Qu'il fasse sens, par exemple dans les sphères biologique et physique, ou bien encore juridique, présente un fort intérêt, car les organisations mettent en jeu des dimensions de cette nature.

\section{Références}

Bachelard G., 1934. Le nouvel esprit scientifique, Paris, PUF. Baechler J., 2008. Agir, Faire, Connaître, Paris, Hermann Philosophie.

Bateson G., 1977. Vers une écologie de l'esprit, tome 1, Paris, Seuil.

Bateson G., 1980. Vers une écologie de l'esprit, tome 2, Paris, Seuil.

Berthoz A., 2003. La décision, Paris, Odile Jacob.

Berthoz A., Debru C., 2015. Anticipation et prédiction. Du geste au voyage mental, Paris, Odile Jacob.

Boltanski L., Chiapello E., 1999. Le nouvel esprit du capitalisme, Paris, Gallimard.

Boutinet J.-P., 2012 [1 ${ }^{\text {re }}$ éd. 1990]. Anthropologie du projet, Paris, PUF.

Boutinet J.-P., Bréchet J.-P., 2014. Logiques de projet, logiques de profit. Convergences ou oppositions, Lyon, Chronique Sociale.

Bratman M.E., 1997. Pour une théorie modeste de l'action planifiée: réponse à Gauthier et Dupuy, in Dupuy J.-P., Livet P. (Eds), Les limites de la rationalité, Paris, La Découverte, 75-88.

Bréchet J.-P., Desreumaux A., 2010. Agir projectif, action collective et autonomie, Management International, 14, 4, 11-23, https://www.erudit.org/fr/revues/mi/2010-v14-n4mi3934/044656ar.pdf.

Bréchet J.-P., Desreumaux, A., 2012. Le projet dans l'action collective, in Allouche J., Encyclopédie des Ressources Humaines, Paris, Vuibert, 1130-1136.

Bréchet J.-P., Emin S., Schieb-Bienfait N., 2014. La rechercheaccompagnement : une pratique légitime, Finance Contrôle Stratégie, 17-2, http://journals.openedition.org/fcs/1477 ; doi: 10.4000/fcs.1477. 
Bréchet J.-P., Gigand G., 2016. Dix ans de transdisciplinarité dans le monde : un bilan instructif, Natures Sciences Sociétés, 24, 1, 67-84, doi: 10.1051/nss/2016003.

Bréchet J.-P., Dufeu I., 2018. Liberté individuelle et force $d u$ collectif, Vulaines-sur-Seine, Éditions du Croquant.

Cometti J.-P., 2011. Qu'est-ce qu'une règle?, Paris, Vrin.

Crozier M., Friedberg E., 1977. L'acteur et le système, Paris, Seuil.

Damasio A., 1995. L'erreur de Descartes: la raison des émotions, Paris, Odile Jacob.

Desreumaux A., Bréchet J.-P., 2009. Quels fondements pour les théories de la firme? Plaidoyer pour une théorie artificialiste de l'action collective fondée sur le projet, in Baudry B., Dubrion B. (Eds), Analyses et transformations de la firme. Une approche pluridisciplinaire, Paris, La Découverte, 61-83.

Desreumaux A., Bréchet J.-P., 2013. L'entreprise comme bien commun, RIMHE, 7, 3, 77-93, doi: 10.3917/ rimhe.007.0077.

Desreumaux A., Bréchet J.-P., 2018. Repenser l'entreprise. Une théorie de l'entreprise fondée sur le Projet, Lille, Presses Universitaires du Septentrion.

Dewey J., 2011. La formation des valeurs, Paris, La Découverte.

Dewey J., 2014 [1 ${ }^{\text {re }}$ éd. 1929]. La quête de certitude. Une étude de la relation entre connaissance et action, Paris, Gallimard.

Dupuy J.-P., Livet P. (Eds), 1997. Les limites de la rationalité. Tome 1: Rationalité, éthique et cognition, Paris, La Découverte.

Emirbayer M., Mische A., 1998. What is Agency?, American Journal of Sociology, 103, 4, 962-1023, https://www.jstor. org/stable/10.1086/231294.

Friedberg E., 1993. Le pouvoir et la règle, Paris, Seuil.

Hatchuel A., 2000. Quel horizon pour les sciences de gestion? Vers une théorie de l'action collective, in David A., Hatchuel A., Laufer R. (Eds), Les nouvelles fondations des sciences de gestion, Paris, Vuibert, 7-34.

Hatchuel A., 2005. Towards an epistemology of collective action: management research as a responsive and action- nable discipline, European Management Review, 2, 1, 3647, doi: 10.1057/palgrave.emr.1500029.

Joas H., 1999. La créativité de l'agir, Paris, Cerf.

Kalberg S., 2010. Les idées, les valeurs et les intérêts. Introduction à la sociologie de Max Weber, Paris, La Découverte/MAUSS, www.revuedumauss.com.fr/media/ KALWEB.pdf.

Lawrence R.J. (Ed.), 2015. Advances in transdisciplinarity 2004-2014, Futures, 65, doi: 10.1016/j.futures.2014.11.007.

Menger P.-M., 2001. Les temps, les causes et les raisons de l'action, in Grenier J.-Y., Grignon C., Menger P.-M. (Eds), Le modèle et le récit, Paris, Éditions de la Maison des Sciences de l'Homme, 103-178.

Morin E., 1977. La méthode 1. La nature de la nature, Paris, Seuil.

Morin E., 1980. La méthode 2. La vie de la vie, Paris, Seuil.

Morin E., 1986. La méthode 3. La connaissance de la connaissance, Paris, Seuil.

Morin E., 2004. La méthode 6. Éthique, Paris, Seuil.

Perroux F., 1973. Pouvoir et économie, Paris, Bordas.

Reynaud J.-D., 1991. Pour une sociologie de la régulation sociale, Sociologies et sociétés, 23, 2, 13-26.

Reynaud J.-D., 1997. Les règles du jeu. L'action collective et la régulation sociale, Paris, A. Colin.

Reynaud J.-D., 2003. Une théorie de la régulation sociale: pour quoi faire?, in de Terssac G. (Ed.), La théorie de la régulation sociale de Jean-Daniel Reynaud. Débats et prolongements, Paris, La Découverte, 399-446.

Reynaud J.-D., 2008. Qu'est-ce qu'une «communauté de projet »?, in Vrancken D., Dubois C., Schoenaers F. (Eds), Penser la négociation. Mélanges en l'honneur de Olgierd Kuty, Bruxelles, De Boeck Supérieur, 189-192.

Schütz A., 1998. Éléments de sociologie phénoménologique, Paris, L'Harmattan.

Simon H., 1959. Theories of decision-making in economics and behavioral science, American Economic Review, 49, 3, 253-283, https://msuweb.montclair.edu/ lebelp/SimonDec TheoryAER1959.pdf.

Sfez L., 1988. La décision, Paris, PUF.

Citation de l'article : Boutinet J.-P., Bréchet J.-P., 2018. Choix, décision et projet dans l'action : préséance du projet dans une perspective pluridisciplinaire. Nat. Sci. Soc. 26, 4, 434-445. 